\title{
Adult Acute Lymphoblastic Leukemia: A Cancer with No Standard of Care
}

\section{Dan Douer}

Leukemia Service, Department of Medicine, Memorial Sloan-Kettering Cancer Center, Weill Cornell Medical College, New York, N.Y., USA

Approximately 6,000 new cases of acute lymphocytic leukemia (ALL) are diagnosed in the USA each year. Most occur in children, but $40 \%$ are in adults 20 years or older. In children, outcomes have improved due to sequential, well-conducted, multi-institutional, randomized clinical trials, rationally designed in a step-by-step manner. Consequently, approximately $80 \%$ of all children with ALL are cured. In contrast, the cure rate of adult ALL has remained at $35-40 \%$ over the past 30 years [1]. Many large multi-institutional studies were also conducted in adults, but most were not developed systematically and very few were randomized. Furthermore, despite variability in patient characteristics between regimens, the outcomes were almost identical. As a result, this rare cancer has no real 'standard-of-care' in adults [2]. In this issue of Acta Haematologica, Buyukasik et al. [3] report a retrospective comparison of 2 treatment approaches in adults.

In general, the treatment of ALL is complex, with a variety of chemotherapy agents used in multiple cycles; all regimens include maintenance and central nervous system prophylaxis which distinguish the treatment of ALL from that of acute myeloid leukemia. With a few exceptions, most regimens can be grouped into 2 fundamentally different models [2]. The first of these, the BerlinFrankfurt-Munster (BFM) model, was developed in pedi- atrics. It has a 2-phase induction. The first phase includes 4 drugs (daunorubicin, prednisone, vincristine and asparaginase), and the second phase contains cyclophosphamide, cytarabine and 6-mercaptopurine. The drugs in the postremission cycles vary within each regimen and between regimens but include asparaginase and a late cycle of delayed reinduction (a truncated modified induction). Hoelzer et al. [4] adopted the BFM model and modified it for adults, and several of the subsequent adult regimens are variants of this model. The second model, the hyperfractionated cyclophosphamide, vincristine, doxorubicin and dexamethasone (hyper-CVAD) regimen, was developed at the MD Anderson Cancer Center. It consists of 2 cycles alternating 4 times for a total of 8 cycles. Cycle A contains fractionated cyclophosphamide, vincristine, doxorubicin and dexamethasone, mostly given in the first 5 days; cycle $\mathrm{B}$ contains high-dose methotrexate and high-dose cytarabine given over 3 days. In contrast to $\mathrm{BFM}$-based regimens, it contains no asparaginase and is used almost only in adults [5]. The structure of 2 alternating cycles is simpler to follow, and it is widely used in the USA. No apparent outcome advantage was reported for either regimen, and the choice of any ALL treatment is generally based on prior training and practice preferences. The role of hematopoietic stem cell transplantation

\section{KARGER}

E-Mail karger@karger.com

www.karger.com/aha
(C) 2013 S. Karger AG, Basel

0001-5792/13/1303-0196\$38.00/0
Dan Douer, MD

Leukemia Service, Department of Medicine, Memorial Sloan-Kettering Cancer Center Weill Cornell Medical College 1275 York Ave, New York, NY 10065 (USA)

E-Mail douerd@mskcc.org 
(HSCT) is also unclear due to conflicting results among clinical trials.

In this issue of Acta Haematologica, Buyukasik et al. [3] report a retrospective analysis of newly diagnosed ALL patients treated at the same 4 institutions in Turkey either with hyper-CVAD (as originally reported in 2000) [5] or one of the commonly used BFM-based regimens [Cancer and Leukemia Group B (CALGB) 8811, reported in 1995] [6]. The overall results highlight the unsatisfactory outcome of adult ALL even among relatively young patients (65 years old or less, median age 30 years). Interestingly, both original studies included older patients. With a combined total of 122 patients (23\% of them undergoing HSCT), 57 (46\%) of them were alive. Most patients achieved a complete remission (CR), i.e. $84 \%$ on hyper-CVAD and $74 \%$ on the CALGB regimen. In other studies, including BFM-based regimens and subsequent reports of hyper-CVAD, the CR rate was 90\% [2]. Importantly, primary resistance with both treatments was uncommon and reported in only $10 \%$ or less of patients. Therefore, a key obstacle in curing adult ALL with current regimens is not the lack of achieving a morphological CR, but rather failing to maintain it, mostly due to relapse. As reported by Buyukasik et al. [3], the cumulative incidence of relapse at 5 years was $45 \%$ for both regimens, indicating a need for more effective, yet safe, approaches than both regimens are currently providing.

An interesting finding of this study was a statistically significant lower overall survival (OS) rate with hyperCVAD compared to the CALGB regimen, with apparently similar patient demographics and disease characteristics, namely a median OS of 16 versus 33 months and 5 -year OS of 26 versus $48 \%$, respectively. In a regression analysis including HSCT and other risk factors, only the treatment type was an independent predictor for OS, with borderline statistical significance. The difference was found to be due to a higher nonrelapse death (NRD) rate with hyper-CVAD of $29 \%$, versus $4 \%$ for the CALGB regimen, mostly due to infections. The authors also compared the OS rates of each regimen to their respective original reports, and for the CALBG regimen they were able to reproduce the OS fairly well. In contrast, the difference in OS was accounted for mostly by a significantly higher NRD rate with hyper-CVAD than reported initially.

These results suggesting that hyper-CVAD could be more toxic in adults compared to one of the commonly used adult BFM-based regimens is intriguing. Both cycles $\mathrm{A}$ and $\mathrm{B}$ of hyper-CVAD contain agents given at doses that are myelotoxic for a total of 8 cycles with a risk of re- current profound neutropenia. Hyper-CVAD treatment was mostly reported from one large single institution, but more recent and smaller retrospective analyses from several centers in Australia reported longer cytopenias than previously reported, though this did not reduce the OS [7]. The higher NRD, mostly from fatal infections, could suggest longer myelotoxicity with hyper-CVAD in the currently reported study. However, a direct comparison of the frequency and duration of treatment-related cytopenias between 2 regimens in the same 4 institutions was not carried out. It is also possible that hyper-CVAD is better tolerated in a single institution compared to its utilization in multiple institutions and the 4 institutions in this report. Differences in managing profound cytopenias between centers may be important, as suggested by Buyukasik et al. [3], who used less comprehensive coverage with prophylactic antimicrobial agents than reported with hyper-CVAD. As opposed to hyper-CVAD, CALGB 8811 was a multi-institutional study, and results from other institutions including the 4 in this report might be more comparable to the original report. BFM-based regimens include asparaginase with unique and potentially fatal toxicities that are avoided in the original hyperCVAD. The authors do not report asparaginase-related unique toxicities, but the NRD rate of $4 \%$ in the CALGB regimen suggests it is less likely that severe asparaginase toxicity was a factor.

These observations reported by Buyukasik et al. [3] are certainly not conclusive, since they are weakened by the retrospective analysis, a relatively small number of patients and the lack of information as to why a specific regimen was chosen. Further, a higher percentage of patients treated by hyper-CVAD underwent HSCT compared to the CALGB regimen, possibly contributing to the lower OS rate. Further, despite the lower OS with hyper-CVAD, it should be noted that the rate of early death in induction was in fact lower compared to the CALGB induction. Nevertheless, this report underscores that close follow-up, prevention and early management is critical in both regimens. However, a randomized trial comparing the 2 regimens as suggested by the authors will not impact clinical practice, mostly since both regimens are unsatisfactory. What is needed are new directions, built on past experience from treating adult and pediatric ALL patients, together with novel concepts. For example, is extensive myelosuppression analogous to acute myeloid leukemia treatment really necessary? Or rather might it be better to increase nonmyelosuppressive agents? This is a concept supported by several observations. Firstly, vincristine and steroids alone induce a CR in half or more 
pediatric and adult patients. In fact, of the 4 drugs in the first induction of a BFM-based regimen, only 1 - daunorubicin - is myelosuppressive. Secondly, all pediatric randomized trials have convincingly shown that longer asparagine depletion by asparaginase increases the cure rate. Thirdly, maintenance therapy with prolonged exposure to nonmyelosuppressive doses of chemotherapy is mandatory for cure, a successful therapeutic approach unique to ALL. Fourthly, several studies in adults including hyper-CVAD [8] and CALGB 19082 [9] showed no outcome improvement by anthracycline intensification and excellent outcome in children with less myelosuppressive regimens. Finally, preliminary results of studies using pediatric regimens, some modified for adults and several based on BFM with less myelosuppressive agents and higher cumulative doses of asparaginase, are encouraging. The most recent CALGB 10403 clinical trial has completed enrollment of young adults to a pediatric protocol, and the results will be informative. In addition, promising novel targeted agents are being introduced in front-line ALL protocols. Within these new concepts, the role of allogeneic HSCT should also be addressed.

For the practicing physician, this report does not provide a clear direction. However, it emphasizes the need for more standard approaches that would be stratified by risk factors that are increasingly becoming more distinctive rather than based on unproven views. The hope is that the wide gap in curing ALL between children and adults (at least young adults) might finally begin to narrow.

\section{References}

$>1$ Pui CH, Evans WE: Treatment of acute lymphoblastic leukemia. N Engl J Med 2006;354: 166-178.

$>2$ Douer D: Is asparaginase a critical component in the treatment of acute lymphoblastic leukemia? Best Pract Res Clin Haematol 2008;21: 647-658.

3 Buyukasik Y, Acar K, Kelkitli E, Uz B, Serefhanoglu B, Ozdemir E, Pamukcuoglu M, Atay H, Bektas O, Turkoz Sucak G, Turgut M, Aksu S, Yagci M, Sayınalp N, Ozcebe OI, Goker H, Haznedaroglu IC: Hyper-CVAD regimen in routine management of adult acute lymphoblastic leukemia: a retrospective multicenter study. Acta Haematol 2013;130:199205.

-4 Hoelzer D, Thiel E, Loffler H, Büchner T, Ganser A, Heil G, Koch P, Freund M, Diedrich $\mathrm{H}$, Ruhl H, Maschmeyer H, Lipp T, Nowrousian MR, Burkert M, Gerecke D, Pralle $H$, Müller U, Lunsken $\mathrm{CH}$, Fülle $\mathrm{H}$, Ho $\mathrm{AD}$, Küchler R, Busch FW, Schneider W, Görg Ch, Emmerich B, Braumann D, Vaupel HA, von Paleske A, Bartels A, Neiss A, Messerer D: Prognostic factors in a multicenter study for treatment of acute lymphoblastic leukemia in adults. Blood 1988;71:123-131.
5 Kantarjian HM, O’Brien S, Smith TL, Cortes J, Giles FJ, Beran M, Pierce S, Huh Y, Andreeff M, Koller C, Ha CS, Keating MJ, Murphy S, Freireich EJ: Results of treatment with hyperCVAD, a dose intensive regimen, in adult acute lymphocytic leukemia. J Clin Oncol 2000; 18:547-561.

6 Larson RA, Dodge RK, Burns CP, Lee EL, Stone RM, Schulman P, Duggan D, Davey FR, Sobol RE, Frankel SR, Hooberman AL, Westbrook CA, Arthur DC, George SL, Bloomfield $\mathrm{CD}$, Schiffer CA: A five-drug remission induction regimen with intensive consolidation for adults with acute lymphoblastic leukemia: cancer and leukemia group B study 8811 . Blood 1995;85:2025-2037.
7 Morris K, Weston H, Mollee P, Marlton P, Gill D, Kenned G: Outcome of treatment of adult acute lymphoblastic leukemia with hyperfractionated cyclophosphamide, doxorubicin, vincristine, dexamethasone/methotrexate, cytarabine: results from an Australian population. Leuk Lymphoma 2011;52:85-89.

8 Thomas DA, O’Brien S, Faderl S, GarciaManero G, Ferrajoli A, Wierda W, Ravandi F, Verstovsek S, Jorgensen JL, Bueso-Ramos C, Andreeff M, Pierce S, Garris R, Keating MJ, Cortes J, Kantarjian HM: Chemoimmunotherapy with a modified hyper-CVAD and rituximab regimen improves outcome in de novo Philadelphia chromosome-negative precursor B-lineage acute lymphoblastic leukemia. J Clin Oncol 2010;28:3880-3889.

$\checkmark$ Stock W, Johnson JL, Stone RM, Kolitz JE, Powell BL, Wetzler M, Westervelt P, Marcucci G, DeAngelo DJ, Vardiman JW, McDonnell D, Mrózek K, Bloomfield CD, Larson RA: Dose intensification of daunorubicin and cytarabine during treatment of adult acute lymphoblastic leukemia: results of Cancer and Leukemia Group B Study 19802. Cancer 2013; 119:90-98. 